\title{
An Image Evaluation System Coupled with Auto-TEM
}

\author{
N. Nakamura*, Y. Fujiyoshi**,***, K. Mitsuoka**, K. Murata*** and T. Shinkawa* \\ *JEOL Ltd., 1-2, Musashino 3-chome, Akishima, Tokyo 196-8558 Japan \\ **Kyoto University, Oiwake-cho, Kitashirakawa, Sakyo-ku, Kyoto 606-8502 Japan \\ ***Agency of Industrial Science and Technology, 2-41-6, Aomi, Koto-ku, Tokyo 135-0064 Japan
}

Computer-aimed automated systems for TEM observation have freed researchers from some laboring routine work on image collection[1][2]. However, the researchers have not yet been released from additional data screening. The researchers have to check images and judge the data quality. Such a job often consumes a lot of time. For the purpose of reducing such laborious work, we have developed a system named PASys, which collects images and evaluates them automatically to detect the object that the system user wants to analyze. We have found that this system can be a great help for membrane protein structure research.

It is not realistic to develop a system applicable to all the kinds of samples, because the object a researcher wants to detect is dependent on the samples and their objectives. A too customized system would also be undesirable since such a system could not be applied to any other case and hence the development cost would be too high. From this point of view, we designed PASys as not one module but united executable modules (Fig.1). IP (Image processing) modules, each of which is specialized to detect particular characteristics in images, can easily be interchanged depending on each case because they are independent of other modules.

The DAQ (data acquisition) module in Fig.1 controls a TEM and collects images according to user-defined parameters. The only thing the user has to do is to tune the TEM ordinarily and to set up some parameters (Table 1). Once the user defines the parameters, the system does the rest of the operation automatically. While the image collection, live movie, current stage position, and number of acquired images are displayed on the PC-C (Fig.1) screen, and the IP modules in Fig.1 evaluate the captured images from the characteristics designed to be detected. After the data acquisition and the image processing, the user can see a list of images sorted by the probability of object existence with the View module (Fig.2).

We developed an IP module for liposome detection to screen samples with two-dimensional crystals of membrane protein. PASys with this IP module astonishingly reduces the operations by giving high evaluated values to the images with liposome. The PASys user can find liposome on the samples easily and efficiently from the values. The user can relocate the sample to the acquired position only by commanding the View module to relocate, and also can capture an image again with higher magnification to check if there is a crystalline area in the liposome with FFT function (Fig.3). 
References

[1] C.S.Potter, et al., Ultramicroscopy 77 (1999), 153-161

[2] G.T.Oostergetel, et al., Ultramicroscopy 74 (1998), 47-59

[3] This research was supported by 2001 NEDO's fund of "Development on Biopolymer Structural Information Utilization Techniques" research project.

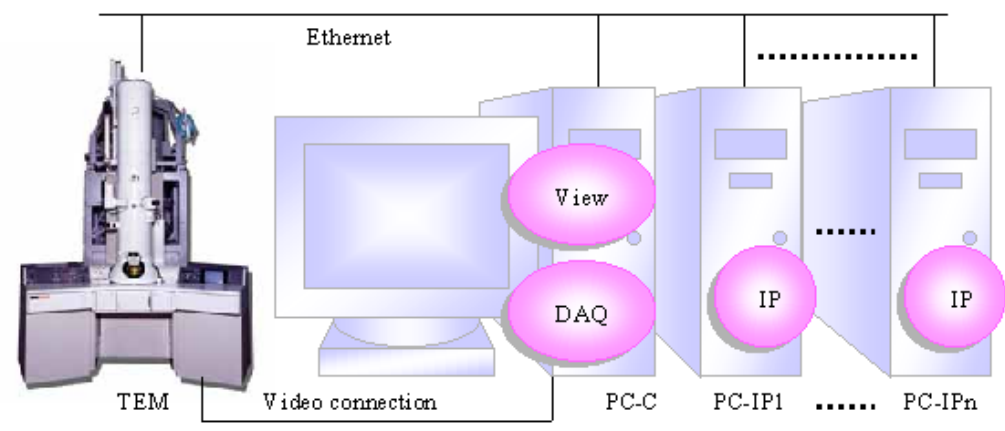

Fig.1. Mechanical connections and software modules of this system. The DAQ module on a computer (PC-C) controls a TEM and captures images. The IP modules on other computers (PC-IP1,.., PC-IPn) analyze the images with specialized image processing algorithms to detect the characteristic features. The View module lists up the images and the results of analysis. It also has some convenient functions like relocation, FFT, etc.

Table1. Examples of parameters on image collection.

\begin{tabular}{l|l}
\hline Parameter & Selectable values \\
\hline Magnification & $\mathrm{x} 1,000, \mathrm{x} 1,500, \mathrm{x} 2,000, \mathrm{x} 2500, \mathrm{x} 3,000, \mathrm{x} 4,000, \mathrm{x} 5,000$, etc. \\
Specimen Number & $1,2,3,4,5$ (when the system is used with a 5-sample holder.) \\
Observation Area & Radius and center position of a circle shape region on each sample. \\
\hline
\end{tabular}

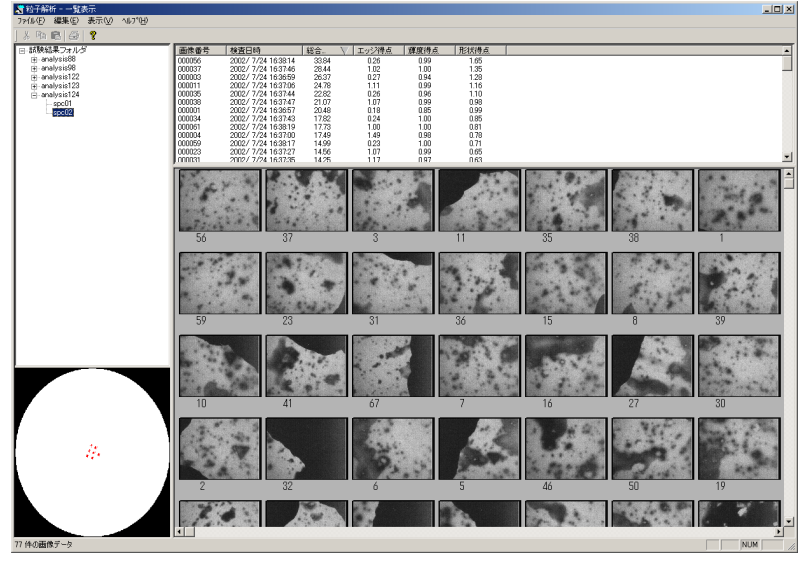

Fig.2. Screen shot of View module showing images and the other data.

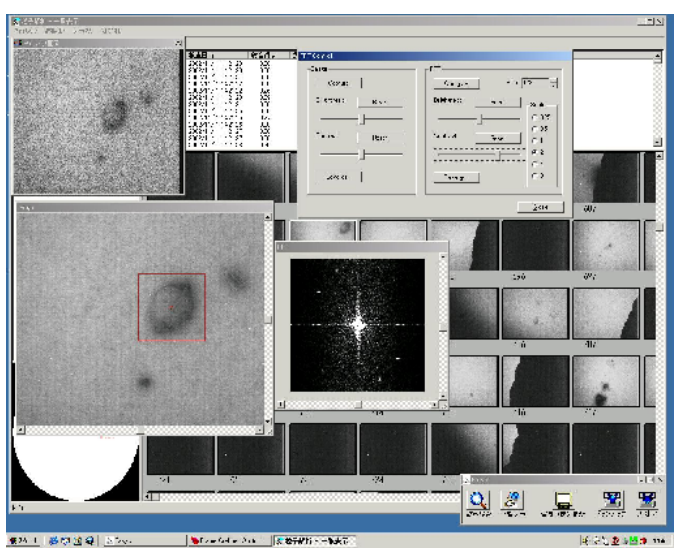

Fig.3. FFT function of the View module. 